\section{Development of a Core Team for the Management of Peripherally Inserted Central Catheters}

To the Editor-We welcome the article by Chopra et al, ${ }^{1}$ in which a systematic review and meta-analysis was carried out to assess the risk of bloodstream infection associated with peripherally inserted central catheters (PICCs) compared with central venous catheters (CVCs) in adults. They examined 1,185 studies and, after including 23 , found a 10 -fold greater risk of central line-associated bloodstream infection (CLABSI) among hospitalized patients (5.2\%) compared with outpatients who received PICCs $(0.5 \%)$. Rates of CLABSI for hospitalized patients who underwent PICC placement were statistically similar to those associated with CVCs. Subsequently, they conclude that the risks and benefits of using PICC lines should be considered before using them in an inpatient setting.

We believe that the findings of this study have important implications for patients, especially as the use of PICCs has increased since the 1990s, and it is reported that they are now the most commonly used CVCs. ${ }^{2,3}$ In our hospital, PICC line insertions are mainly carried out by interventional radiologists, and the number of insertions has increased from 92 in 2002 to 741 in 2012, which includes both planned services (eg, oncology, outpatient parenteral antibiotic therapy) and unplanned services (eg, pediatrics, surgery, medicine, orthopedics). Because PICCs have previously been considered to be less of a risk for infection, we collected baseline data on CLABSI due to PICCs through a retrospective observational study. 4,5 We included all inpatients who had a PICC line inserted over a 12-month period between 2011 and 2012. All microbiology culture results of these patients for 12 weeks after insertion of the PICC line were retrieved retrospectively. We defined confirmed CLABSI as a recognized pathogen from at least 1 positive blood culture (peripheral, line, or both) and a positive quantitative line tip culture for the same organism, with no other site positive for the same organism. Alternatively, it was also defined as a recognized pathogen from at least 1 peripheral culture and the same organism in at least 1 line culture in cases where line tip was negative or not done, with no other site positive for the same organism. A probable CLABSI was defined as follows: a recognized pathogen from at least 1 line culture and negative peripheral blood culture and negative line tip culture or peripheral cultures and line tip culture not done, with no other site positive for the same organism. The total number of PICCs inserted during the study period was 534 from 434 patients. In 74 patients, there were multiple insertions between 2 and 7 lines. We looked at mean and median time to infection in confirmed and probable line infections and organisms causing line infections (Table 1). We found the confirmed PICC line infection rate in our cohort to be $2.62 \%$ (confidence interval, 1.27\%-3.97\%). Limitations of our study, however, include small patient numbers, retrospective collection of data, and limited analysis of patient characteristics.

We agree with Chopra et al $^{1}$ that switching to PICC lines in place of insertion of CVCs alone would not be an effective way of reducing CLABSI in hospitalized patients. Chopra et al have suggested that PICC line guidelines on suitable placement and maintenance checklists should be developed. Currently, our medical doctors have no formal training in the insertion and care of PICC lines, and this may result in lack of or delayed recognition of complications. A recent study found that doctors' knowledge of indications for PICC line insertion is poor, and one-third of doctors never examine for exit site infection. ${ }^{6}$ We believe that a core PICC line team, initially employing experienced nursing staff from the interventional radiology service, could be developed to improve management of PICC lines and prevent complications, such as thromboemobolism and infection. The team could carry out daily ward rounds, providing surveillance of lines and training for staff in inserting PICCs if clinically indicated. With regular updates, medical teams could become more confident in inserting and managing PICC lines safely and effectively on the wards, reducing the risk of CLABSI.

\section{ACKNOWLEDGMENTS}

Potential conflicts of interest. All authors report no conflicts of interest relevant to this article. All authors submitted the ICMjE Form for Disclosure of Potential Conflicts of Interest, and the conflicts that the editors consider relevant to this article are disclosed here.

TA B LE 1. Mean and Median Time to Infection in Confirmed and Probable Line Infections and Organisms Causing Line Infections

\begin{tabular}{lcccccc}
\hline $\begin{array}{l}\text { Line } \\
\text { infection }\end{array}$ & $\begin{array}{c}\text { Mean (range), } \\
\text { days }\end{array}$ & $\begin{array}{c}\text { Median, } \\
\text { days }\end{array}$ & Enterobacteriaceae & MSSA & $\begin{array}{c}\text { Coagulase-negative } \\
\text { Staphylococci }\end{array}$ & $\begin{array}{c}\text { Candida } \\
\text { spp. }\end{array}$ \\
\hline Confirmed & $25.1(8-55)$ & 20 & 5 & 4 & 3 & 2 \\
Probable & $36(13-69)$ & 26 & 2 & 0 & 1 & 0 \\
\hline
\end{tabular}

NOTE. Data are no. of organisms, unless otherwise indicated. MSSA, meticillin-sensitive Staphylococcus aureus. 


\section{Benjamin J. Parcell, FRCPath; \\ Lasantha Ratnayake, FRCPath; Ian Zealley, FRCP, FRCR; ${ }^{3}$ Gabby Phillips, FRCPath ${ }^{1}$}

Affiliations: 1. Medical Microbiology, Ninewells Hospital and Medical School, National Health Service Tayside, Dundee DD1 9SY, Scotland, United Kingdom; 2. Department of Medical Microbiology, James Paget University Hospitals National Health Service Foundation Trust, Lowestoft Road, Gorleston-on-Sea, Norfolk NR31 6LA, England, United Kingdom; 3. Department of Radiology, Ninewells Hospital, Dundee DD1 9SY, Scotland, United Kingdom.

Address correspondence to Benjamin Parcell, FRCPath, Medical Microbiology, Ninewells Hospital and Medical School, National Health Service Tayside, Dundee DD1 9SY, Scotland, United Kingdom (b.parcell@nhs.net). Infect Control Hosp Epidemiol 2013;34(12):1328-1329

(C) 2013 by The Society for Healthcare Epidemiology of America. All rights reserved. 0899-823X/2013/3412-0017\$15.00. DOI: $10.1086 / 673996$

\section{REFERENCES}

1. Chopra V, O'Horo JC, Rogers MAM, Maki DG, Safdar N. The risk of bloodstream infection associated with peripherally inserted central catheters compared with central venous catheters in adults: a systematic review and meta-analysis. Infect Control Hosp Epidemiol 2013;34:908-918.

2. Gabriel J. Peripherally inserted central catheters: expanding UK nurses' practice. $B r$ I Nurs 1996;5:71-74.

3. Chopra V, Flanders SA, Saint $S$. The problem with peripherally inserted central catheters. JAMA 2012;308:1527-1528.

4. Al Raiy B, Fakih MG, Bryan-Nomides N, et al. Peripherally inserted central venous catheters in the acute care setting: a safe alternative to high-risk short-term central venous catheters. Am $J$ Infect Control 2010;38:149-153.

5. Gunst M, Matsushima K, Vanek S, et al. Peripherally inserted central catheters may lower the incidence of catheter-related blood stream infections in patients in surgical intensive care units. Surg Infect (Larchmt) 2011;12:279-282.

6. Chopra V, Kuhn L, Coffey CE Jr, et al. Hospitalist experiences, practice, opinions, and knowledge regarding peripherally inserted central catheters: a Michigan survey. J Hosp Med 2013;8:309-314.

\section{Reply to Parcell et al}

To the Editor-We thank Parcell et al $^{1}$ for their kind words regarding our recent article. ${ }^{2}$ The authors share their findings from a retrospective cohort analysis of hospitalized patients finding infection rates that (when taking into account the confidence intervals) do not appear dissimilar to our analysis. While the authors do mention some limitations, however, they neglect to include denominator data so as to express infections per catheter day, descriptions as to how peripherally inserted central catheters (PICCs) were used (inpatient only vs inpatient and outpatient), and whether losses to followup occurred in their cohort. These data would have been useful to make robust conclusions about the relative risk of central line-associated bloodstream infection (CLABSI) with PICCs compared with other types of central venous catheters.
How best to move forward to improve PICC care in hospitalized settings remains an important question. On the one hand, it is clear that these devices play important roles, and simply removing them from the armamentarium of venous access in hospitalized patients is not logical or wise. However, on the other hand, it is also becoming more apparent that these devices pose a considerable risk of CLABSI, and inattention to this risk can result in adverse outcomes. ${ }^{3,4}$ As pointed out by Parcell et $\mathrm{al},{ }^{1}$ insertion practices represent just the tip of the iceberg when it comes to these events. Meticulous attention to site care, device management, and prompt removal of PICCs that are clinically no longer warranted are cornerstones to the prevention of downstream complications. ${ }^{5}$ How best to leverage existing resources to attain this important, longer-term objective is unknown. While the authors suggest that a core PICC team involving interventional radiologists may prove valuable, PICC insertions in the United States have largely become the purview and practice of specially trained, vascular access nurses who have made significant advances in venous access. ${ }^{6}$ At many centers (ours included), vascular nurses provide insertion and the majority of subsequent care (scheduled dressing changes, line troubleshooting) for PICCs. Given this backdrop, the feasibility of having highly trained radiologists to assist with PICC care by conducting daily ward rounds and surveillance of lines remains debatable.

Nevertheless, the point brought forth by these authors is well taken. A homogenous care team is a critical aspect in the battle against PICC complications, and several local and institutional reports of such success can be found in the peerreviewed literature. ${ }^{7,8}$ These reports share three common themes: defining which practices are most valuable, standardizing these care processes, and consolidating monitoring and benchmarking efforts. In the United States, the Infusion Nursing Society and the Association for Vascular Access have initiated and developed standards of practice that have influenced policy and practice for vascular access specialists. ${ }^{9}$ While the Infusion Nurses Society guidelines are an important advance, their diffusion across domains to other providers (eg, physicians) and dissimilar settings (eg, the United Kingdom) may represent important barriers. Multidisciplinary collaborations engaging all relevant stakeholders-physicians, interventional radiologists, nursing staff, infection preventionists, patients, and institutional leadership-may represent the best approach forward.

\section{ACKNOWLEDGMENTS}

Potential conflicts of interest. All authors report no conflicts of interest relevant to this article. All authors submitted the ICMJE Form for Disclosure of Potential Conflicts of Interest, and the conflicts that the editors consider relevant to this article are disclosed here.

Vineet Chopra, MD, MSc; ${ }^{1}$ Nasia Safdar, $\mathrm{MD}, \mathrm{PhD}^{2}$ 